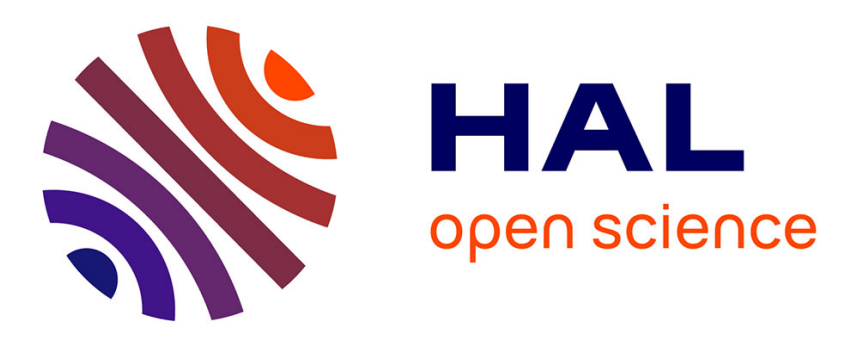

\title{
Late Ventilator-Induced Diaphragmatic Dysfunction After Extubation
}

Haikel Dridi, Boris Jung, Mohamad Yehya, Aurelien Daurat, Steven Reiken, Johan Moreau, Andrew Marks, Stefan Matecki, Alain Lacampagne, Samir Jaber

\section{To cite this version:}

Haikel Dridi, Boris Jung, Mohamad Yehya, Aurelien Daurat, Steven Reiken, et al.. Late Ventilator-Induced Diaphragmatic Dysfunction After Extubation. Critical Care Medicine, In press, 10.1097/CCM.0000000000004569 . hal-02956989

\section{HAL Id: hal-02956989 \\ https://hal.science/hal-02956989}

Submitted on 14 Oct 2020

HAL is a multi-disciplinary open access archive for the deposit and dissemination of scientific research documents, whether they are published or not. The documents may come from teaching and research institutions in France or abroad, or from public or private research centers.
L'archive ouverte pluridisciplinaire HAL, est destinée au dépôt et à la diffusion de documents scientifiques de niveau recherche, publiés ou non, émanant des établissements d'enseignement et de recherche français ou étrangers, des laboratoires publics ou privés. 


\title{
Late Ventilator-Induced Diaphragmatic Dysfunction After Extubation
}

\author{
Haikel Dridi, $\mathrm{PhD}^{1,2}$; Boris Jung, $\mathrm{MD}, \mathrm{PhD}^{1,3}$; Mohamad Yehya, $\mathrm{PhD}^{1}$; Aurelien Daurat, $\mathrm{MD}, \mathrm{MSc}^{4,5}$; \\ Steven Reiken, $\mathrm{PhD}^{2}$; Johan Moreau, $\mathrm{MD}^{1}$; Andrew R. Marks, $\mathrm{MD}, \mathrm{PhD}^{2}$; Stefan Matecki, $\mathrm{MD}, \mathrm{PhD}^{1,5}$; \\ Alain Lacampagne, $\mathrm{PhD}^{1}$; Samir Jaber, $\mathrm{MD}, \mathrm{PhD}^{1,4}$
}

Objectives: Mechanical ventilation is associated with primary diaphragmatic dysfunction, also termed ventilator-induced diaphragmatic dysfunction. Studies evaluating diaphragmatic function recovery after extubation are lacking. We evaluated early and late recoveries from ventilator-induced diaphragmatic dysfunction in a mouse model.

Design: Experimental randomized study.

Setting: Research laboratory.

Subjects: C57/BL6 mice.

Interventions: Six groups of C57/BL6 mice. Mice were ventilated for 6 hours and then euthanatized immediately $(n=18)$, or 1 $(n=18)$ or 10 days after extubation with $(n=5)$ and without $S 107$ $(n=16)$ treatment. Mice euthanatized immediately after 6 hours of anesthesia $(n=15)$ or after 6 hours of anesthesia and 10 days of recovery $(n=5)$ served as controls.

Measurements and Main Results: For each group, diaphragm force production, posttranslational modification of ryanodine receptor, oxidative stress, proteolysis, and cross-sectional areas were evaluated. After 6 hours of mechanical ventilation, diaphragm force production was decreased by $25-30 \%$, restored to the control levels 1 day after extubation, and secondarily decreased by $20 \%$ 10 days after extubation compared with controls. Ryanodine receptor was protein kinase A-hyperphosphorylated, S-nitrosylated, oxidized, and depleted of its stabilizing subunit calstabin-1 6 hours after the onset of the mechanical ventilation, 1 and 10 days after

${ }^{1}$ PhyMedExp, Montpellier University, INSERM, CNRS, Montpellier, France.

${ }^{2}$ Department of Physiology and Cellular Biophysics, Clyde and Helen Wu Center for Molecular Cardiology Columbia University College of Physicians and Surgeons, New York, NY.

${ }^{3}$ Medical Intensive Care Unit, Montpellier University and Montpellier University Health Care Center, Montpellier, France.

${ }^{4}$ Saint Eloi Department of Anesthesiology and Critical Care Medicine, Montpellier University and Montpellier University Health Care Center, Montpellier, France.

${ }^{5}$ Arnaud de Villeneuve Physiological Department, Montpellier University and Montpellier University Health Care Center, Montpellier, France.

Copyright (c) 2020 by the Society of Critical Care Medicine and Wolters Kluwer Health, Inc. All Rights Reserved. extubation. Post extubation treatment with S107, a Rycal drug that stabilizes the ryanodine complex, did reverse the loss of diaphragmatic force associated with mechanical ventilation. Total protein oxidation was restored to the control levels 1 day after extubation. Markers of proteolysis including calpain 1 and calpain 2 remained activated 10 days after extubation without significant changes in cross-sectional areas.

Conclusions: We report that mechanical ventilation is associated with a late diaphragmatic dysfunction related to a structural alteration of the ryanodine complex that is reversed with the $\mathrm{S} 107$ treatment. (Crit Care Med 2020; XX:00-00)

Key Words: mechanical ventilation; ryanodine receptor calcium release channel; Rycal; ventilator-induced diaphragmatic dysfunction; weaning

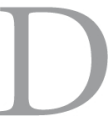
iaphragm weakness is associated with the loss of diaphragm force production and is observed up to $64 \%$ of patients upon ICU admission (1). Sepsis and mechanical ventilation (MV) are the two main known risk factors of diaphragm weakness $(1,2)$. The majority of patients with limb muscle weakness do have diaphragm weakness but up to $67 \%$ of patients without limb muscle weakness may be diagnose with diaphragm weakness which contributes to underrecognition of diaphragm weakness in ICU patients (3).

Diaphragm weakness is associated with poor outcome, that is, difficult weaning $(4,5)$ and prolonged ICU stay $(1,4,5)$. Since it may affect respiratory function and dependence on ventilatory support, and it may participate in late reintubation, recovery from diaphragm weakness deserves particular attention. However, recovery from diaphragm weakness in the ICU has been sparsely studied. In one cohort of 43 patients, 23 presented diaphragm weakness at admission, where nine of them showed signs of recovery during the ICU stay (6). Along with sepsis and shock, the use of MV by itself leads to diaphragm weakness within a few hours $(7,8): 12$ hours in rats (9) and 18 hours in critically ill patients (10). This specific weakness linked with MV has been coined ventilator-induced diaphragmatic dysfunction (VIDD) by Vassilakopoulos and Petrof (11). 
When spontaneous breathing is abolished, VIDD is the consequence of diaphragm unloading and ventilated patient biopsies show signs of sarcomeric disruptions (10), fatty infiltration (12), proteolysis, and autophagy (13), leading to atrophy $(10,14,15)$ and mitochondrial oxidative stress (16). Atrophy has been associated with the loss of diaphragmatic force production in numerous animals and in a few human studies $(10,17)$. Loss of force production with no atrophy, a condition coined dynapenia, is observed in a 6 hours MV mouse model $(7,8)$ and has been suggested in humans $(18,19)$. Early diaphragmatic dynapenia during MV is linked to excitation-contraction coupling (ECC) impairment as a consequence of structural remodeling of the type $1 \mathrm{Ca}^{2+}$ release channel/ ryanodine receptor 1 (RyR1) $(8,16)$. Posttranslational modifications including protein kinase A (PKA)-hyperphosphorylation, cysteine oxidation, and nitrosylation dissociate the stabilizing subunit FK506 binding protein 12 (FKBP12) (e.g., calstabin 1) from the $\mathrm{Ca}^{2+}$ release channel. RyR1 remodeling, in turn, results in impaired $\mathrm{Ca}^{2+}$ handling due to pathologic $\mathrm{Ca}^{2+}$ leak from the sarcoplasmic reticulum to the cytosol and is associated with ECC impairment and muscle weakness $(8,16)$. Although the deleterious impact of MV on diaphragm structure and function has been established, only two studies have evaluated the diaphragmatic function recovery after MV discontinuation ( 9 , 20). Two animal studies have reported a complete recovery in diaphragmatic force production 7 hours to 24 hours after the end of MV, with no data later in the course of recovery $(9,20)$.

Since prolonged diaphragmatic dysfunction may have an impact on respiratory function, reintubation, and ICU readmission, we aimed to study diaphragmatic structure and function early ( $1 \mathrm{~d})$ and late $(10 \mathrm{~d})$ after MV discontinuation in a mouse model.

\section{MATERIALS AND METHODS}

\section{Murine Model of VIDD}

The online supplement (Supplemental Digital Content 1, http:// links.lww.com/CCM/F806) contains details regarding ethics approval, diaphragm function evaluation, and the agents used. The present experimental work conforms to the guidelines laid out in the Guide for the Care and Use of Laboratory Animals (www. nap.edu/readingroom/books/labrats) and was approved by the local animal care ethic committee. We used the same study protocol that has been previously described by our group $(7,8)$. Six groups of adult C57/BL6 mice (12 wk old) were constituted: a control group (euthanasia after anesthesia $n=15$ ), MV for 6 hours (MV, $n=18$ ), 1 day of recovery following 6 hours of MV (MV $+24 \mathrm{hr}, n=18), 10$ days of recovery following 6 hours of MV with and without S107 (MV + $10 d+S 107, n=5 ; M V+10$ $\mathrm{d}, n=16$ ), and a control group with 10 days recovery from anesthesia (control $+10 \mathrm{~d}, n=5$ ). All in vivo and ex vivo experiments were performed by investigators blinded to treatment groups.

\section{Diaphragm Function}

Mice were euthanized by exsanguination and the entire diaphragm was surgically excised. Isometric contractile properties were assessed as described elsewhere (7). The excised diaphragm strip was mounted into jacketed tissue bath chambers filled with equilibrated and oxygenated Krebs solution. The muscles were supramaximally stimulated using square wave pulses (Model S48; Grass Instruments, Aurora Scientific Inc, Aurora, ON, Canada). The force-frequency relationship was determined by sequentially stimulating the muscles for $600 \mathrm{~ms}$ at $10,20,30,50,60,80,100$, and $120 \mathrm{~Hz}$ with 1 minute between each stimulation train (7). Muscle fatigue was assessed by measuring the loss of force in response to repeated stimuli (30 $\mathrm{Hz}, 300 \mathrm{~ms}$ duration) during 5 minutes as previously reported (7). After measurement of contractile properties, muscles were measured at Lo (the length at which the muscle produced maximal isometric tension), dried, and weighted. For comparative purposes, muscle force production was normalized for total muscle strip cross-sectional area and expressed in $\mathrm{N} / \mathrm{cm}^{2}$. The total muscle strip cross-sectional area was determined by dividing muscle weight by its length and tissue density $(1.056 \mathrm{~g} /$ $\left.\mathrm{cm}^{3}\right)$.

\section{Immunoprecipitation}

RyR1 was immunoprecipitated from diaphragm using an antiRyR1-specific antibody $(2 \mu \mathrm{g})$ in $0.5 \mathrm{~mL}$ of a modified radioimmune precipitation assay buffer $(50 \mathrm{~mm}$ Tris- $\mathrm{HCl}, \mathrm{pH} 7.2$, $0.9 \% \mathrm{NaCl}, 5.0 \mathrm{~mm} \mathrm{NaF}, 1.0 \mathrm{~mm} \mathrm{Na}_{3} \mathrm{Vo}_{4}, 1 \%$ Triton X-100, and protease inhibitors) overnight at $4^{\circ} \mathrm{C}$. RyR1-specific antibody was RyR1-1327, an affinity-purified rabbit polyclonal antibody raised against a KLH-conjugated peptide with the amino acid sequence CAEPDTDYENLRRS, corresponding to residues 1,327-1,339 of mouse skeletal RyR1, with an additional cysteine residue added to the amino terminus and affinity purified with the unconjugated peptide. The immune complexes were incubated with protein A-Sepharose beads (Sigma, St. Louis, $\mathrm{MO}$ ) at $4^{\circ} \mathrm{C}$ for 1 hour, and the beads were washed three times with radioimmune precipitation assay buffer. The immunoprecipitates were size-fractionated on sodium dodecyl sulfatepolyacrylamide gel electrophoresis gels (4-20\% for RyR1 and calstabin 1) and transferred onto nitrocellulose membranes for 2 hours at $200 \mathrm{~mA}$. Immunoblots were developed using the following primary antibodies: anti-RyR1 (1:2,000; Affinity Bioreagents, Golden, CO), antiphospho-RyR-Ser(P) (1:5,000; Affinity Bioreagents), anti-calstabin (FKBP12 C-19, 1:1,000; Santa Cruz Biotechnology, Santa Cruz, CA), and antibody against cysteine nitrosylation (1:1,000; Sigma). To determine channel oxidation, the carbonyl groups in the protein side chains were derivatized to 2,4-dinitrophenol (DNP) by reaction with 2,4-dinitrophenylhydrazine. The DNP signal associated with RyR1 was determined using a specific anti-DNP antibody according to the manufacturer's instructions (Millipore, Billerica, MA). All immunoblots were developed using an Odyssey system (LI-COR Biosciences, Lincoln, NE) with infrared Dy-labeled anti-mouse and anti-rabbit $\operatorname{IgG}(1: 10,000$ dilution) secondary antibodies.

\section{Western Blots}

Immunoblot analysis was performed to evaluate the ratios of the active (cleaved) forms of calpain 1,2 and caspase 3 to total 
levels of calpain 1,2 and caspase 3 isoforms, respectively. Total protein ubiquitination was evaluated using polyubiquitinylated conjugates antibody as previously described (21). The ratio of LC3/2 to LC3/1 was evaluated as a marker of autophagy as previously reported (22).

Total protein oxidation was measured using an oxyblot kit (Millipore). The carbonyl groups in the protein side chains were derivatized to DNP by reaction with 2,4-dinitrophenylhydrazine. The DNP signal associated with oxidized proteins was determined using a specific anti-DNP antibody according to the manufacturer's instructions (Millipore). GAPDH was used as a loading control. We used primary antibodies as follows: caspase 3 (1:1,000; Abcam Ab13585, Cambridge, MA) and GAPDH (1:2,500; Abcam Ab9485). Calpain 1 (1:1,000; Abcam Ab108400), calpain 2 (1:1,000; Abcam Ab39165), LC3/1-2 (1:1,000; Abcam Ab51520), and ubiquitination (Abcam Ab7780). Secondary antibodies were anti-rabbit $800 \mathrm{~nm}$ and anti-mouse $700 \mathrm{~nm}(1: 10,000)$.

\section{Histology}

Cross-sectional area was determined using cryosections $(10 \mu \mathrm{m})$ and myosin ATPase histochemical staining as previously described $(7,8,23)$. Muscle membrane was counterstained with dystrophin antibodies as previously reported (16). The musclefiber cross-sectional area was determined using the ImageJ analysis software (https://imagej.nih.gov/ij/download.html) and expressed in $\mu \mathrm{m}^{2}$ ( $\sim 900$ fibers per group were analyzed). Muscle fibrosis was evaluated using Masson trichrome staining.

\section{Statistical Analysis}

Data were presented as means and SES unless otherwise indicated. When more than two groups were compared, one- or two-way analysis of variances or Kruskal-Wallis tests (according to normality) were used. The $\alpha$ level was set at 0.05 , corrected for multiple comparisons with the Holm-Sidak test, and was bilaterally tested. Statistical analysis was performed using GraphPad Prism 6.0 (San Diego, CA). The number of mice per group required to identify differences in major physiologic parameters was estimated based on previous experience with this model.

\section{RESULTS}

\section{Six Hours of MV in Mice Is Associated With 10 Days Late VIDD}

MV is associated with diaphragm dysfunction in a murine model of VIDD. To investigate the mechanisms involved in the recovery from VIDD, sarcoplasmic reticulum fractions from ventilated diaphragm during 6 hours, ventilated diaphragm during 6 hours followed by 1 or 10 days recovery and controls were purified to analyze the composition of RyR1 macromolecular complex and posttranslational modifications known to be associated with channel leak as a proximal mechanism involved in VIDD $(8,16)$. MV diaphragms exhibit PKA-hyperphosphorylation, oxidation, and cysteine nitrosylation and were depleted of calstabin compared with controls (EFig. 1A-E, Supplemental
Digital Content 2, http://links.lww.com/CCM/F807; legend, Supplemental Digital Content 1, http://links.lww.com/CCM/ F806). Indeed, this called "biochemical signature of leaky RyR1" was persistent 1 and 10 days after MV (EFig. 1 A-E, Supplemental Digital Content 2, http://links.lww.com/CCM/F807; legend, Supplemental Digital Content 1, http://links.lww.com/ CCM/F806). Indeed, diaphragm function, evaluated by measuring the ex vivo-specific force production, was significantly decreased after 6 hours of MV and 10 days after MV compared with the controls (EFig. 1F-J, Supplemental Digital Content 2, http://links.lww.com/CCM/F807; legend, Supplemental Digital Content 1, http://links.lww.com/CCM/F806). Of note, transitory enhancement of the diaphragm force production 1 day after MV was observed (EFig. $1 \mathrm{H}-J$, Supplemental Digital Content 2, http://links.lww.com/CCM/F807; legend, Supplemental Digital Content 1, http://links.lww.com/CCM/F806). Diaphragm fatigue resistance was significantly decreased by 6 hours of MV and 10 days after extubation (EFig. $1 K$, Supplemental Digital Content 2, http://links.lww.com/CCM/F807; legend, Supplemental Digital Content 1, http://links.lww.com/ CCM/F806).

\section{Late Diaphragm Dysfunction Is Associated With Activation of $\mathrm{Ca}^{2+}$ Proteases (Calpain 1 and 2) Without Atrophy or Fibrosis}

In order to investigate the mechanism responsible of the late diaphragm dysfunction observed in our model beside the impairment of the $\mathrm{Ca}^{2+}$ homeostasis, we evaluated the activity of the two major $\mathrm{Ca}^{2+}$ activated proteases, calpain 1 and 2 . Calpain 1 was significantly increased 1 and 10 days after extubation compared with the controls while calpain 2 was significantly increased only 10 days after extubation (EFig. $2 A-C$, Supplemental Digital Content 3, http://links.lww.com/CCM/ F808; legend, Supplemental Digital Content 1, http://links.lww. com/CCM/F806). Indeed, a transitory activation of caspase 3 (EFig. 2A-D, Supplemental Digital Content 3, http://links. lww.com/CCM/F808; legend, Supplemental Digital Content 1, http://links.lww.com/CCM/F806), autophagy (EFig. 2A-E, Supplemental Digital Content 3, http://links.lww.com/CCM/F808; legend, Supplemental Digital Content 1, http://links.lww.com/ CCM/F806), and proteins oxidation (EFig. 2A-G, Supplemental Digital Content 3, http://links.lww.com/CCM/F808; legend, Supplemental Digital Content 1, http://links.lww.com/CCM/ F806) were observed only after 6 hours of MV. Total proteins ubiquitination was unchanged (EFig. $2 A-F$, Supplemental Digital Content 3, http://links.lww.com/CCM/F808; legend, Supplemental Digital Content 1, http://links.lww.com/CCM/F806).

To investigate the histological changes related to $\mathrm{Ca}^{2+}$ release impairment and calpains activation, we measured cross-sectional areas and indices of fibrosis among our groups using immunohistochemistry and Masson trichrome staining on $10 \mu \mathrm{m}$ diaphragm cryosections. Diaphragm cross-sectional areas were comparable among the group without significant changes of the fibers subtype (EFig. 3A-C, Supplemental Digital Content 4, http://links.lww.com/CCM/F809; legend, SupplementalDigitalContent1,http://links.lww.com/CCM/F806). 
Indeed, indices of fibrosis were unchanged among the groups (EFig. 3, D and E, Supplemental Digital Content 4, http:// links.lww.com/CCM/F809; legend, Supplemental Digital Content 1, http://links.lww.com/CCM/F806).

\section{Rycals Drug S107 Prevents the Calstabin 1 Dissociation From RyR1 and Improves Diaphragmatic Force Production}

In order to rescue the late diaphragm dysfunction observed 10 days after extubation, we treated our ventilated mice with S107, a Rycal drug which prevents calstabin 1 depletion from RyR1 and reduces pathologic $\mathrm{Ca}^{2+}$ leak from the sarcoplasmic reticulum. S107 significantly increased calstabin 1 binding to RyR1 despite the persistence of channel oxidation, phosphorylation, and nitrosylation (EFig. $4 A-E$, Supplemental Digital Content 5, http://links.lww.com/CCM/F810; legend, Supplemental Digital Content 1, http://links.lww.com/CCM/F806). S107 also prevented mice from late diaphragm dysfunction 10 days after extubation (EFig. 4F-J, Supplemental Digital Content 5, http://links.lww.com/CCM/F810; legend, Supplemental Digital Content 1, http://links.lww.com/CCM/F806).

\section{DISCUSSION}

We report for the first time in a murine model of VIDD that diaphragmatic dysfunction induced by 6 hours of MV is associated with early recovery, but with late diaphragmatic dysfunction observed 10 days after extubation. Late diaphragmatic dysfunction was associated with RyR1 complex structural remodeling and was reversed by the orally administered Rycal drug S107, a RyR1 complex stabilizer.

Delayed extubation failure leading to reintubation can occur up to $7-10$ days after initial weaning success $(24,25)$, diaphragmatic dysfunction being one of the major mechanisms (26). During critical illness, diaphragmatic dysfunction can result from the consequences of sepsis, muscle unloading, medications, and any other condition associated with ICU acquired weakness (27). Primary diaphragmatic dysfunction can also be related to MV, a condition known as VIDD (11), which has deleterious impact on diaphragm structure, mass $(10,15,23,28)$, and force production $(10,13,14)$. Different patterns of diaphragmatic function during critical illness have been described (6), and loss of force production is mitigated by spontaneous breathing $(4,19,29)$. Despite two animal studies which have reported an early recovery from VIDD after MV discontinuation $(9,20)$, long-term follow up studies are scarce.

Our results suggest that even after early recovery from MV, intracellular events persist and are associated with late diaphragmatic dysfunction. We herein report that RyR1 structural remodeling combining oxidation, nitrosylation, inadequate phosphorylation, and calstabin unbound persist up 10 days after MV discontinuation (EFig. 1A-E, Supplemental Digital Content 2, http://links.lww.com/CCM/F807; legend, Supplemental Digital Content 1, http://links.lww.com/CCM/ F806). Maladaptive cAMP-dependent protein kinase A mediated phosphorylation and redox-dependent modifications (nitrosylation and oxidation) of RyR1 have been linked to the depletion of the stabilizing unit calstabin 1 from the RyR 1 channel complex $(30,31)$. RyR1 channel complex remodeling and instability result in impaired $\mathrm{Ca}^{2+}$ handling with a pathologic sarcoplasmic reticulum $\mathrm{Ca}^{2+}$ leak associated with diaphragmatic contractile dysfunction and other conditions such as muscle dystrophy, chronic muscle fatigue, and aging. RyR1 channel complex remodeling is the consequence of burst in oxidative stress (EFig. 2, Supplemental Digital Content 3, http://links.lww.com/CCM/F808; legend, Supplemental Digital Content 1, http://links.lww.com/CCM/F806) (12, 3537) and adrenergic activation of the protein kinase A (EFig. $\mathbf{5}$, Supplemental Digital Content 6, http://links.lww.com/CCM/ F870; legend, Supplemental Digital Content 1, http://links.lww. com/CCM/F806), which lead to actin-myosin uncoupling (8), proteolysis, and autophagy activation (EFig. 2, Supplemental Digital Content 3, http://links.lww.com/CCM/F808; legend, Supplemental Digital Content 1, http://links.lww.com/CCM/ F806). Calpain 1 and 2, calcium-dependent proteases implicated in cleaving myofilament proteins, have been associated with diaphragmatic dysfunction $(7,27,38)$ and long-term myocardial remodeling post myocardial infarction $(39,40)$. Calpain 1 and 2 levels were increased 10 days after extubation, possibly secondary both to the oxidative stress burst and the RyR1 sarcoplasmic reticulum calcium leak (EFig 3, Supplemental Digital Content 4, http://links.lww.com/CCM/ F809; and EFig. 4, Supplemental Digital Content 5, http://links. lww.com/CCM/F810 [legend, Supplemental Digital Content 1, http://links.lww.com/CCM/F806]) (8).

In the present study, we observed a transient diaphragmatic force recovery 1 day after MV discontinuation despite the presence of RyR1 remodeling. One hypothesis to explain this transient recovery could be that the mitochondria network may have had the buffering capacity to prevent cytosolic $\mathrm{Ca}^{2+}$ overload at the early stage (1 d after extubation) (41) but that its capacity might have been insufficient at a later stage ( $10 \mathrm{~d}$ after extubation).

The 6 hours MV mouse model is not associated with histological changes (EFig 4, Supplemental Digital Content 5, http://links.lww.com/CCM/F810; legend, Supplemental Digital Content 1, http://links.lww.com/CCM/F806), a finding in accordance with a previous study using the same model (8) and with the moderate correlation between force production and mass (42). Force production depends on muscle mass and also on other factors such as the fiber-to-muscle-length ratio, the intracellular calcium concentration, the amount of myosin heads available for interaction with actin filaments, or the ECC (43).

The persistence of RyR1 dysfunction after 10 days of recovery reinforces the possibility of a pharmacological approach with molecules such as Rycals that are small drug molecules stabilizing the RyR1 which have been previously reported to prevent VIDD in mice (8). In the present study, the use of S107, a Rycal administered in drinking water from MV discontinuation to day 10 was associated with calstabin rebind to RyR1 with no effect on oxidation, nitrosylation, and phosphorylation (EFig. 3, Supplemental Digital Content 4, http://links.lww.com/CCM/F809; legend, Supplemental Digital Content 1, http://links.lww.com/CCM/F806). The use 
of S107 was moreover associated with a complete force production recovery 10 days after MV discontinuation (EFig. 3, Supplemental Digital Content 4, http://links.lww.com/CCM/ F809; legend, Supplemental Digital Content 1, http://links. lww.com/CCM/F806) potentially paving the way toward a treatment for VIDD.

The present study has certain limitations. First, we have evaluated day 1 and day 10 time points and do not present more time points. Second, it would have been of interest to further explore the mechanisms associated with delayed diaphragmatic dysfunction following MV such as the mitochondrial calcium buffering capacity, but this was beyond the scope of this study, which focused on diaphragm function.

\section{CONCLUSIONS}

We report for the first time that 6 hours of MV secondarily impairs diaphragmatic function despite a transient recovery 24 hours after extubation. Significant diaphragmatic dysfunction occurs 10 days after MV discontinuation and extubation and is associated with a persistent RyR1 remodeling that is reversible with the use of the orally available S107, a Rycal drug. Further exploration of the mechanisms associated with late diaphragmatic failure and studies in humans and whether S107 would be a drug candidate to treat VIDD are warranted.

Drs. Dridi and Jung contributed equally to this work.

Drs. Dridi, Jung, Reiken, Marks, Lacampagne, and Jaber designed experiments, analyzed data, and edit/wrote the article. Drs. Yehya, Daurat, Reiken, and Moreau designed experiments and analyzed data.

Supplemental digital content is available for this article. Direct URL citations appear in the printed text and are provided in the HTML and PDF versions of this article on the journal's website (http://journals.Iww.com/ ccmjournal).

Supported, in part, by INSERM, Region Languedoc Roussillon, Montpellier-Nîmes Medical School, and Association Française contre les Myopathies.

Dr. Marks received funding from ARMGO (Tarrytown, NY), a company developing compounds targeting ryanodine receptor and have patents on Rycals (Columbia University); he received support for article research from the National Institutes of Health; and he disclosed off-label product use of Rycal. The remaining authors have disclosed that they do not have any potential conflicts of interest.

For information regarding this article, E-mail: s-jaber@chu-montpellier.fr

\section{REFERENCES}

1. Demoule A, Jung $B$, Prodanovic $H$, et al: Diaphragm dysfunction on admission to the intensive care unit. Prevalence, risk factors, and prognostic impact-a prospective study. Am J Respir Crit Care Med 2013; 188:213-219

2. Supinski GS, Callahan LA: Diaphragm weakness in mechanically ventilated critically ill patients. Crit Care 2013; 17:R120

3. Dres M, Dubé BP, Mayaux J, et al: Coexistence and impact of limb muscle and diaphragm weakness at time of liberation from mechanical ventilation in medical intensive care unit patients. Am J Respir Crit Care Med 2017; 195:57-66

4. Jung $B$, Moury $\mathrm{PH}$, Mahul $\mathrm{M}$, et al: Diaphragmatic dysfunction in patients with ICU-acquired weakness and its impact on extubation failure. Intensive Care Med 2016; 42:853-861

5. Dres M, Schmidt M, Ferre A, et al: Diaphragm electromyographic activity as a predictor of weaning failure. Intensive Care Med 2012; 38:2017-2025
6. Demoule A, Molinari N, Jung B, et al: Patterns of diaphragm function in critically ill patients receiving prolonged mechanical ventilation: A prospective longitudinal study. Ann Intensive Care 2016; 6:75

7. Mrozek S, Jung B, Petrof BJ, et al: Rapid onset of specific diaphragm weakness in a healthy murine model of ventilator-induced diaphragmatic dysfunction. Anesthesiology 2012; 117:560-567

8. Matecki S, Dridi H, Jung B, et al: Leaky ryanodine receptors contribute to diaphragmatic weakness during mechanical ventilation. Proc Nat/ Acad Sci U S A 2016; 113:9069-9074

9. Thomas D, Maes K, Agten A, et al: Time course of diaphragm function recovery after controlled mechanical ventilation in rats. $J$ App/ Physiol (1985) 2013; 115:775-784

10. Jaber $S$, Petrof $B J$, Jung $B$, et al: Rapidly progressive diaphragmatic weakness and injury during mechanical ventilation in humans. $A m ~ J$ Respir Crit Care Med 2011; 183:364-371

11. Vassilakopoulos T, Petrof BJ: Ventilator-induced diaphragmatic dysfunction. Am J Respir Crit Care Med 2004; 169:336-341

12. Picard $M$, Jung $B$, Liang $F$, et al: Mitochondrial dysfunction and lipid accumulation in the human diaphragm during mechanical ventilation. Am J Respir Crit Care Med 2012; 186:1140-1149

13. Hussain SN, Mofarrahi M, Sigala I, et al: Mechanical ventilationinduced diaphragm disuse in humans triggers autophagy. $\mathrm{Am} J$ Respir Crit Care Med 2010; 182:1377-1386

14. Levine S, Nguyen T, Taylor N, et al: Rapid disuse atrophy of diaphragm fibers in mechanically ventilated humans. N Engl J Med 2008; 358:1327-1335

15. Powers SK, Kavazis AN, Levine S: Prolonged mechanical ventilation alters diaphragmatic structure and function. Crit Care Med 2009; 37:S347-S353

16. Dridi $H$, Yehya $M$, Barsotti $R$, et al: Mitochondrial oxidative stress induces leaky ryanodine receptor during mechanical ventilation. Free Radic Biol Med 2020; 146:383-391

17. van den Berg M, Hooijman PE, Beishuizen A, et al: Diaphragm at rophy and weakness in the absence of mitochondrial dysfunction in the critically ill. Am J Respir Crit Care Med 2017; 196:1544-1558

18. Goligher EC, Fan E, Herridge MS, et al: Evolution of diaphragm thickness during mechanical ventilation. Impact of inspiratory effort. $\mathrm{Am} J$ Respir Crit Care Med 2015; 192:1080-1088

19. Kim WY, Suh HJ, Hong SB, et al: Diaphragm dysfunction assessed by ultrasonography: Influence on weaning from mechanical ventilation. Crit Care Med 2011; 39:2627-2630

20. Bruells CS, Bergs I, Rossaint R, et al: Recovery of diaphragm function following mechanical ventilation in a rodent model. PLoS One 2014; 9:e87460

21. Yoshihara T, Ichinoseki-Sekine N, Kakigi R, et al: Repeated exposure to heat stress results in a diaphragm phenotype that resists ventilator-induced diaphragm dysfunction. J Appl Physiol (1985) 2015; 119:1023-1031

22. Klionsky DJ, Abdelmohsen K, Abe A, et al: Guidelines for the use and interpretation of assays for monitoring autophagy (3rd edition). Autophagy 2016; 12:1-222

23. Jung B, Constantin JM, Rossel N, et al: Adaptive support ventilation prevents ventilator-induced diaphragmatic dysfunction in piglet: An in vivo and in vitro study. Anesthesiology 2010; 112:1435-1443

24. Thille AW, Boissier F, Ben Ghezala $H$, et al: Risk factors for and prediction by caregivers of extubation failure in ICU patients: A prospective study. Crit Care Med 2015; 43:613-620

25. Ruan SY, Teng NC, Wu HD, et al: Durability of weaning success for liberation from invasive mechanical ventilation: An analysis of a nationwide database. Am J Respir Crit Care Med 2017; 196:792-795

26. Thille AW, Richard JC, Brochard L: The decision to extubate in the in tensive care unit. Am J Respir Crit Care Med 2013; 187:1294-1302

27. Jaber S, Jung B, Matecki S, et al: Clinical review: Ventilator-induced diaphragmatic dysfunction-human studies confirm animal model findings! Crit Care 2011; 15:206

28. Yang L, Luo J, Bourdon J, et al: Controlled mechanical ventilation leads to remodeling of the rat diaphragm. Am J Respir Crit Care Med 2002; $166: 1135-1140$

29. DiNino E, Gartman EJ, Sethi JM, et al: Diaphragm ultrasound as a predictor of successful extubation from mechanical ventilation. Thorax $2014 ; 69: 423-427$ 
30. Reiken S, Lacampagne A, Zhou $\mathrm{H}$, et al: PKA phosphorylation activates the calcium release channel (ryanodine receptor) in skeletal muscle: Defective regulation in heart failure. J Cell Biol 2003; 160:919-928

31. Bellinger AM, Reiken S, Carlson C, et al: Hypernitrosylated ryanodine receptor calcium release channels are leaky in dystrophic muscle. Nat Med 2009; 15:325-330

32. Andersson DC, Betzenhauser MJ, Reiken S, et al: Ryanodine receptor oxidation causes intracellular calcium leak and muscle weakness in aging. Cell Metab 2011; 14:196-207

33. Bellinger AM, Reiken S, Carlson C, et al: Hypernitrosylated ryanodine receptor calcium release channels are leaky in dystrophic muscle. Nat Med 2009; 15:325-330

34. Bellinger AM, Mongillo M, Marks AR: Stressed out: The skeletal muscle ryanodine receptor as a target of stress. J Clin Invest 2008; 118:445-453

35. Jaber S, Sebbane M, Koechlin C, et al: Effects of short vs. prolonged mechanical ventilation on antioxidant systems in piglet diaphragm. Intensive Care Med 2005; 31:1427-1433

36. Kavazis AN, Talbert EE, Smuder AJ, et al: Mechanical ventilation induces diaphragmatic mitochondrial dysfunction and increased oxidant production. Free Radic Biol Med 2009; 46:842-850
37. McClung JM, Kavazis AN, Whidden MA, et al: Antioxidant administration attenuates mechanical ventilation-induced rat diaphragm muscle atrophy independent of protein kinase B (PKB Akt) signalling. J Physiol 2007; 585:203-215

38. Powers SK, Wiggs MP, Sollanek KJ, et al: Ventilator-induced diaphragm dysfunction: Cause and effect. Am J Physiol Regul Integr Comp Physiol 2013; 305:R464-R477

39. Ye T, Wang Q, Zhang Y, et al: Over-expression of calpastatin inhibits calpain activation and attenuates post-infarction myocardial remodeling. PLoS One 2015; 10:e0120178

40. Wan F, Letavernier E, Le Saux CJ, et al: Calpastatin overexpression impairs postinfarct scar healing in mice by compromising reparative immune cell recruitment and activation. Am J Physiol Heart Circ Physio/ 2015; 309:H1883-H1893

41. Parks RJ, Murphy E, Liu JC: Mitochondrial permeability transition pore and calcium handling. Methods Mol Biol 2018; 1782:187-196

42. Jung $B$, Nougaret $S$, Conseil $M$, et al: Sepsis is associated with a preferential diaphragmatic atrophy: A critically ill patient study using tridimensional computed tomography. Anesthesiology 2014; 120:1182-1191

43. Sieck GC, Ferreira LF, Reid MB, et al: Mechanical properties of respiratory muscles. Compr Physiol 2013; 3:1553-1567 